\title{
Acceleration methods for series: A probabilistic perspective
}

\author{
José A. Adell and Alberto Lekuona
}

\begin{abstract}
We introduce a probabilistic perspective to the problem of accelerating the convergence of a wide class of series, paying special attention to the computation of the coefficients, preferably in a recursive way. This approach is mainly based on a differentiation formula for the negative binomial process which extends the classical Euler's transformation. We illustrate the method by providing fast computations of the logarithm and the alternating zeta functions, as well as various real constants expressed as sums of series, such as Catalan, Stieltjes, and Euler-Mascheroni constants.
\end{abstract}

Mathematics Subject Classification (2010). Primary 11Y60, 60E05; Secondary 33B15, 41A35.

Keywords. Series acceleration, negative binomial process, alternating zeta function, Catalan constant, Stieltjes constants, Euler-Mascheroni constant.

\section{Introduction}

Suppose that a certain function or real constant is given by a series of the form

$$
\sum_{j=0}^{\infty} f(j) r^{j}, \quad 0<r<1 .
$$

From a computational point of view, it is not only important that the geometric rate $r$ is a rational number as small as possible, but also that the coefficients $f(j)$ are easy to compute, since the series in (1.1) can also be written as

$$
\sum_{j=0}^{\infty}(f(2 j)+r f(2 j+1)) r^{2 j},
$$

The authors are partially supported by Research Projects DGA (E-64), MTM2015-67006$\mathrm{P}$, and by FEDER funds. 
thus improving the geometric rate from $r$ to $r^{2}$. Obviously, this procedure can be successively implemented (see formula (2.8) in Section 2).

The aim of this paper is to give a probabilistic perspective to the problem of accelerating the convergence of a wide class of series, paying special attention to the computation of the coefficients, preferably in a recursive way. This approach is mainly based on the differential calculus for the negative binomial process developed in [4].

More precisely, denote by $\mathbb{Z}_{+}$the set of nonnegative integers and by $\mathbb{N}=\mathbb{Z}_{+} \backslash\{0\}$. The $m$ th forward differences of any function $\phi: \mathbb{Z}_{+} \rightarrow \mathbb{C}$ are recursively defined by $\Delta^{0} \phi(j)=\phi(j), \Delta^{1} \phi(j)=\phi(j+1)-\phi(j), j \in \mathbb{Z}_{+}$, and

$$
\Delta^{m} \phi(j)=\Delta^{1}\left(\Delta^{m-1} \phi\right)(j)=\sum_{k=0}^{m}\left(\begin{array}{c}
m \\
k
\end{array}\right)(-1)^{m-k} \phi(j+k),
$$

for any $m \in \mathbb{N}$ and $j \in \mathbb{Z}_{+}$. On the other hand, for any $k \in \mathbb{N}$, consider the negative binomial process $\left(Z_{k}(s)\right)_{s \geq 0}$ defined as

$$
P\left(Z_{k}(s)=j\right)=\left(\begin{array}{c}
k-1+j \\
k-1
\end{array}\right)\left(\frac{s}{s+1}\right)^{j} \frac{1}{(s+1)^{k}}, \quad s \geq 0, \quad j \in \mathbb{Z}_{+} .
$$

Finally, let $S$ be a nonnegative random variable independent of each negative binomial process $\left(Z_{k}(s)\right)_{s \geq 0}, k \in \mathbb{N}$, such that $E S^{m}<\infty, m \in \mathbb{N}$, where $E$ stands for mathematical expectation. For any function $\phi: \mathbb{Z}_{+} \rightarrow \mathbb{C}$ and $k \in \mathbb{N}$, it has been shown in [4, Theorem 8.1] the following Taylor's expansion formula

$$
\begin{aligned}
& \sum_{m=0}^{\infty}\left(\begin{array}{c}
k-1+m \\
k-1
\end{array}\right) \Delta^{m} \phi(0) E S^{m}=E \phi\left(Z_{k}(S)\right) \\
& =\sum_{j=0}^{\infty}\left(\begin{array}{c}
k-1+j \\
k-1
\end{array}\right) \phi(j) E \frac{S^{j}}{(S+1)^{k+j}},
\end{aligned}
$$

whenever the series under consideration are convergent. Borisov and Shkodrov [10] have shown (1.3) when the random variable $S$ is replaced by a real number $x \in(-1,1]$, thus extending the classical Euler's series transformation for $k=x=1$ (c.f. Abramowitz and Stegun [1, p.16]).

In this paper, we will use formula (1.3) with $k=1$ to compute the Stieltjes constants (Section 3), particularly the Euler-Mascheroni constant (Section 4). The more restrictive choice $k=1$ and $\phi(j)=E(1-2 T)^{j}, j \in \mathbb{Z}_{+}$, for some random variable $T$ taking values in $[0,1]$ will be used in Section 2 to compute inverse moments of $T$. Depending on the choice of $T$, we can efficiently compute analytic functions such as logarithms and the alternating zeta function, as well as certain real constants expressed as sums of series, such as the Catalan constant.

Finally, we mention that formula (1.3) is one example of the differential calculus for linear operators represented by stochastic processes developed in [4]. As we will see in the following sections, this probabilistic methodology gives a unified approach to deal with various problems in analytic number theory. In this regard, differentiation formulas for linear operators represented 
by gamma processes have been used in [2] to obtain asymptotic estimates for Stieltjes constants, as well as to compute each Stieltjes constant in the classical spirit of Stieltjes and Berndt [9] (c.f. [3]). In many occasions, such a probabilistic approach gives short proofs of the results under consideration. For instance, the concavity of Dirichlet's eta and beta functions shown in [7] (an analytical proof of the same result can be found in Alzer and Kwong [8]).

\section{Computation of inverse moments}

Many functions and real constants allow for a probabilistic representation of the form $E(1+a T)^{-1}, a>-1$, where $T$ is a random variable taking values in $[0,1]$. Such inverse moments can be computed by means of a series having a geometric rate of convergence, as shown in the following theorem. This result could essentially be derived from (1.3) by setting $k=1, \phi(j)=E(1-2 T)^{j}$, $j \in \mathbb{Z}_{+}$, and $S=a / 2, a \geq 0$. However, we give here a direct elementary proof of it.

Theorem 2.1. Let $T$ be a random variable taking values in $[0,1]$. For any $a>-1$, we have

$$
E \frac{1}{1+a T}=\frac{2}{a+2} \sum_{j=0}^{\infty} E(1-2 T)^{j}\left(\frac{a}{a+2}\right)^{j} .
$$

Proof. Let $t \in[0,1]$ and $a>-1$. Since

$$
\frac{|1-2 t||a|}{a+2}<1
$$

we have

$$
\frac{2}{a+2} \sum_{j=0}^{\infty}(1-2 t)^{j}\left(\frac{a}{a+2}\right)^{j}=\frac{1}{1+a t} .
$$

The conclusion follows from (2.2), replacing $t$ by the random variable $T$ and then taking expectations.

Theorem 2.1 is close in spirit to the linear acceleration methods for alternating series developed by Cohen et al. [15], Borwein [11], and Coffey [12], among other authors. Such methods use a sequence of polynomials such as Chebyshev, Laguerre, and other polynomials $P_{j}(x)$ with $P_{j}(-1) \neq 0, j \in \mathbb{Z}_{+}$, to compute the coefficients, and produce in specific examples better geometric rates than that in (2.1). In this regard, the underlying sequence of polynomials in $(2.1)$ is $P_{j}(x)=(1-2 x)^{j}, j \in \mathbb{Z}_{+}$. This choice, however, is interesting for various reasons. In first place, because the coefficients $E(1-2 T)^{j}, j \in \mathbb{Z}_{+}$, can be recursively computed, as shown in the following examples. Secondly, because the random variable $1-2 T$ takes values in $[-1,1]$ and this implies that $E(1-2 T)^{j}$ converges to 0 as $j \rightarrow \infty$, although slowly, in general. Finally, if $T$ is a symmetric random variable around $1 / 2$, the odd coefficients vanish and the geometric rate in $(2.1)$ improves from $a /(a+2)$ to $a^{2} /(a+2)^{2}$. This is the case, for instance, for the logarithm function considered below. 
Let $U$ and $V$ be two independent random variables having the uniform distribution on $[0,1]$. We consider the following coefficients

$$
c(j)=E\left(1-2 U^{2}\right)^{j}, \quad d(j)=E\left(1-2 V^{2} U^{2}\right)^{j}, \quad j \in \mathbb{Z}_{+} .
$$

Such coefficients appear in Examples 1-2 below and can be recursively evaluated as follows.

Lemma 2.2. For any $j \in \mathbb{Z}_{+}$, we have

$$
(2 j+3) c(j+1)=(-1)^{j+1}+2(j+1) c(j)
$$

and

$$
(2 j+3) d(j+1)=c(j+1)+2(j+1) d(j) .
$$

Proof. Let $v \in[0,1]$. Using integration by parts, we have

$$
\begin{aligned}
& E\left(1-2 v^{2} U^{2}\right)^{j+1}=\int_{0}^{1}\left(1-2 v^{2} u^{2}\right)^{j+1} d u \\
& =\left(1-2 v^{2}\right)^{j+1}+2(j+1) \int_{0}^{1} 2 v^{2} u^{2}\left(1-2 v^{2} u^{2}\right)^{j} d u \\
& =\left(1-2 v^{2}\right)^{j+1}+2(j+1)\left(E\left(1-2 v^{2} U^{2}\right)^{j}-E\left(1-2 v^{2} U^{2}\right)^{j+1}\right) .
\end{aligned}
$$

Both recursive equalities in Lemma 2.2 follow by choosing $v=1$ and $v=V$ in (2.4), respectively.

Numerical computations show that $c(j)$ and $d(j)$ tend to 0 , as $j \rightarrow \infty$, at the rate $1 / \sqrt{j}$.

\section{Example 1: The logarithm function.}

Apart from its own interest, a fast computation of the logarithm function will be needed to evaluate the Stieltjes constants (see Section 3) and, particularly, the Euler-Mascheroni constant (Section 4).

Corollary 2.3. For any $a>-1$, we have

$$
\begin{aligned}
\frac{\log (1+a)}{a} & =\frac{2}{a+2} \sum_{j=0}^{\infty} \frac{1}{2 j+1}\left(\frac{a}{a+2}\right)^{2 j} \\
& =\frac{4(a+2)}{a^{2}} \sum_{j=0}^{\infty}(-1)^{j} c(j)\left(\frac{a^{2}}{a^{2}+8(a+1)}\right)^{j+1} .
\end{aligned}
$$

Proof. Let $U$ be a random variable having the uniform distribution on $[0,1]$. Observe that

$$
E(1-2 U)^{2 j+1}=0, \quad E(1-2 U)^{2 j}=E U^{2 j}=\frac{1}{2 j+1}, \quad j \in \mathbb{Z}_{+} .
$$

We therefore have from Theorem 2.1

$$
\frac{\log (1+a)}{a}=E \frac{1}{1+a U}=\frac{2}{a+2} \sum_{j=0}^{\infty} \frac{1}{2 j+1}\left(\frac{a}{a+2}\right)^{2 j}
$$


Set $b=-a^{2} /(a+2)^{2}>-1$. Using (2.6), (2.7), and Theorem 2.1, we get

$$
\begin{aligned}
\frac{\log (1+a)}{a} & =\frac{2}{a+2} \sum_{j=0}^{\infty} E U^{2 j}\left(\frac{a}{a+2}\right)^{2 j}=\frac{2}{a+2} E \frac{1}{1+b U^{2}} \\
& =\frac{2}{a+2} \frac{2}{b+2} \sum_{j=0}^{\infty} E\left(1-2 U^{2}\right)^{j}\left(\frac{b}{b+2}\right)^{j} \\
& =\frac{4}{b(a+2)} \sum_{j=0}^{\infty} E\left(1-2 U^{2}\right)^{j}\left(\frac{b}{b+2}\right)^{j+1} .
\end{aligned}
$$

This, together with (2.3), completes the proof.

The first equality in (2.5) is well known (c.f. Abramowitz and Stegun [1, p. 68]). Observe that the second series in (2.5) produces faster computations of the logarithm, specially when $a$ is close to 0 , without paying an extra computational cost, since the coefficients $c(j)$ can be recursively evaluated. For instance, if $a=1 / 4$, the geometric rates of both series in (2.5) are 1/81 and $1 / 161$, respectively. On the other hand, starting from the first equality in $(2.5)$, we get for any $a>-1$ and $l=0,1,2, \ldots$

$$
\frac{\log (1+a)}{a}=\frac{2}{a+2} \sum_{j=0}^{\infty} f_{l}(j)\left(\frac{a}{a+2}\right)^{j 2^{l+1}},
$$

where

$$
f_{l}(j)=\sum_{k=0}^{2^{l}-1} \frac{1}{j 2^{l+1}+2 k+1}\left(\frac{a}{a+2}\right)^{2 k} .
$$

The same could be done starting from the second equality in (2.5). This shows that geometric rates can be considerably improved at the price of complicating the coefficients under consideration.

\section{Example 2: The Catalan constant.}

Among the various equivalent definitions of the Catalan constant $K$, we give the following

$$
K=\sum_{j=0}^{\infty} \frac{(-1)^{j}}{(2 j+1)^{2}} .
$$

With the help of the coefficients $d(j)$ defined in (2.3), we give the following fast computation of $K$.

Corollary 2.4. We have

$$
K=\frac{2}{3} \sum_{j=0}^{\infty} d(j) \frac{1}{3^{j}}
$$


Proof. Let $U$ and $V$ be two independent random variables having the uniform distribution on $[0,1]$. By (2.6) and Theorem 2.1, we see that

$$
K=\sum_{j=0}^{\infty} E\left(-(U V)^{2}\right)^{j}=E \frac{1}{1+(U V)^{2}}=\frac{2}{3} \sum_{j=0}^{\infty} E\left(1-2 V^{2} U^{2}\right)^{j} \frac{1}{3^{j}}
$$

which, in conjunction with (2.3), completes the proof.

Different fast computations of $K$ are known (c.f. Karatsuba [20], Kh. and T. Hessami Pilehrood [17], and the references therein). In [17], for instance, the authors compute $K$ by means of a series with the geometric rate $2^{-10}$. The coefficients of such a series are more involved than the coefficients $d(j)$ in (2.9), as they depend on a quotient of polynomials $p(j) / q(j)$ whose degrees are 6 and 8, respectively.

\section{Example 3: The alternating zeta function.}

This function is defined as

$$
\eta(z)=\sum_{m=0}^{\infty} \frac{(-1)^{m}}{(m+1)^{z}}, \quad \Re(z)>0,
$$

or, using a Newton series expansion (c.f. Flajolet and Vepstas [16]), as

$$
\eta(z)=\sum_{n=0}^{\infty} \Delta^{n} \eta(0)\left(\begin{array}{l}
z \\
n
\end{array}\right), \quad \Re(z)>0, \quad \eta(0)=\frac{1}{2} .
$$

For any function $g: \mathbb{Z}_{+} \rightarrow \mathbb{C}$, consider the discrete Cesàro operator given by

$$
C g(j)=\frac{1}{j+1} \sum_{k=0}^{j} g(k), \quad j \in \mathbb{Z}_{+},
$$

and denote by $C^{n}$ the $n$th iterate of $C, n \in \mathbb{Z}_{+}\left(C^{0}=I\right)$. As we will see in Theorem 2.5 below, such iterates appear in the coefficients of certain series providing fast computations of $\eta$.

We recall here the probabilistic representations of the iterates $C^{n} g(j)$ shown in [6]. Let $\left(U_{k}\right)_{k \geq 1}$ be a sequence of independent identically distributed random variables having the uniform distribution on $[0,1]$ and let $\left(V_{k}\right)_{k>1}$ be an independent copy of $\left(U_{k}\right)_{k \geq 1}$. Denote by $1_{A}$ the indicator function of the set $A$. For each $j \in \mathbb{N}$, the random variable

$$
S_{j}(t)=\sum_{k=1}^{j} 1_{[0, t]}\left(U_{k}\right), \quad 0 \leq t \leq 1 \quad\left(S_{0}(t) \equiv 0\right)
$$

has the binomial law with parameters $j$ and $t$, that is,

$$
P\left(S_{j}(t)=k\right)=\left(\begin{array}{l}
j \\
k
\end{array}\right) t^{k}(1-t)^{j-k}, \quad k=0,1, \ldots, j .
$$

On the other hand, denote by $T_{n}=V_{1} \cdots V_{n}, n \in \mathbb{N}$. It has been shown in $[6$, Theorem 2.3] the representation

$$
C^{n} g(j)=E g\left(S_{j}\left(T_{n}\right)\right), \quad j \in \mathbb{Z}_{+}, \quad n \in \mathbb{N} .
$$


In particular, if $g(j)=(-1)^{j}, j \in \mathbb{Z}_{+}$, we have from (2.13) and (2.14)

$$
C^{n} g(j)=E(-1)^{S_{j}\left(T_{n}\right)}=\left(E(-1)^{S_{1}\left(T_{n}\right)}\right)^{j}=E\left(1-2 T_{n}\right)^{j},
$$

for any $j \in \mathbb{Z}_{+}$and $n \in \mathbb{N}$. Using these notations, we give the following.

Corollary 2.5. Let $g(j)=(-1)^{j}, j \in \mathbb{Z}_{+}$. For any $n \in \mathbb{Z}_{+}$, we have

$$
\eta(n)=\frac{2}{3} \sum_{j=0}^{\infty} C^{n} g(j) \frac{1}{3^{j}}
$$

and

$$
\left.\Delta^{n} \eta(0)=\frac{2}{3} \sum_{j=0}^{\infty}(C-I)^{n} g(j) \frac{1}{3^{j}} \quad\left((C-I)^{0}=I\right)\right) .
$$

Proof. Theorem 2.5 is true for $n=0$, as follows from (2.11). Assume that $n \in \mathbb{N}$. Since $E T_{n}^{m}=E V_{1}^{m} \cdots E V_{n}^{m}=(m+1)^{-n}, m \in \mathbb{Z}_{+}$, we have from (2.10) and Theorem 2.1

$$
\begin{aligned}
\eta(n)=\sum_{m=0}^{\infty}(-1)^{m} E T_{n}^{m}=E \frac{1}{1+T_{n}} & =\frac{2}{3} \sum_{j=0}^{\infty} E\left(1-2 T_{n}\right)^{j} \frac{1}{3^{j}} \\
& =\frac{2}{3} \sum_{j=0}^{\infty} C^{n} g(j) \frac{1}{3^{j}},
\end{aligned}
$$

where the last equality follows from (2.15). Using (1.2), (2.16), and Fubini's theorem, we get

$$
\Delta^{n} \eta(0)=\sum_{k=0}^{n}\left(\begin{array}{l}
n \\
k
\end{array}\right)(-1)^{n-k} \eta(k)=\frac{2}{3} \sum_{j=0}^{\infty}\left(\sum_{k=0}^{n}\left(\begin{array}{l}
n \\
k
\end{array}\right)(-1)^{n-k} C^{k} g(j)\right) \frac{1}{3^{j}} .
$$

This shows (2.17) and completes the proof.

By (2.11) and (2.17), we can evaluate $\eta(z), \Re(z)>0$, using a Newton series expansion whose coefficients $\Delta^{n} \eta(0)$ can be computed by means of a series with geometric rate $1 / 3$. In turn, the coefficients of such a series, namely, $(C-I)^{n} g(j)$ can be computed in a recursive way. In this respect, it should be mentioned that Borwein [11] and Coffey [12] obtained efficient algorithms to compute $\eta(z)$ having the form

$$
\sum_{j=0}^{n-1} \frac{c_{j}}{(j+1)^{z}}
$$

where the coefficients $c_{j}$ are built up from Chebyshev, Laguerre, and other polynomials $P_{j}(x)$ satisfying the condition $P_{j}(-1) \neq 0, j \in \mathbb{Z}_{+}$. 


\section{The Stieltjes constants}

Consider the Riemann zeta function

$$
\zeta(z)=\sum_{m=0}^{\infty} \frac{1}{(m+1)^{z}}, \quad \Re(z)>1 .
$$

Recall that

$$
(z-1) \zeta(z)=1+\sum_{n=0}^{\infty} \frac{(-1)^{n} \gamma_{n}}{n !}(z-1)^{n+1}, \quad z \in \mathbb{C},
$$

where the coefficients $\left(\gamma_{n}\right)_{n \geq 0}$ are known as Stieltjes constants $\left(\gamma_{0}\right.$ is the Euler-Mascheroni constant). Some ingredients will be needed to compute such constants. In first place, the Bernoulli numbers appearing in the expansion

$$
\frac{z}{1-e^{-z}}=\sum_{k=0}^{\infty} \frac{(-1)^{k} B_{k}}{k !} z^{k}, \quad|z|<2 \pi .
$$

Secondly, the derivatives $\left((-1)^{k} \alpha_{k}\right)_{k \geq 0}$ of the alternating zeta function at 1 . In other words,

$$
\eta(z)=\sum_{k=0}^{\infty} \frac{(-1)^{k} \alpha_{k}}{k !}(z-1)^{k}, \quad \Re(z)>0,
$$

where

$$
\alpha_{k}=\sum_{m=0}^{\infty}(-1)^{m} \frac{\log ^{k}(m+1)}{m+1}, \quad k \in \mathbb{Z}_{+} \quad\left(\alpha_{0}=\log 2\right) .
$$

Finally, the "geometric" rates $(r(j))_{j \geq 0}$ defined as

$$
r(j)=E \frac{U^{j}}{(U+1)^{j+1}}, \quad j \in \mathbb{Z}_{+},
$$

where $U$ is a random variable having the uniform distribution on $[0,1]$. In fact, the following auxiliary result can be easily shown using integration by parts.

Lemma 3.1. The rates $(r(j))_{j \geq 0}$ can be recursively evaluated as

$$
r(0)=\log 2, \quad r(j)=\log 2-\sum_{k=0}^{j-1} \frac{1}{(k+1) 2^{k+1}}<\frac{1}{(j+1) 2^{j}}, \quad j \in \mathbb{N} .
$$

We will need the following two technical facts. On the one hand, let $U$ and $T$ be two independent random variables such that $U$ is uniformly distributed on $[0,1]$ and $T$ has the exponential density $\rho(\theta)=e^{-\theta}, \theta \geq 0$. Using Fubini's theorem (see also [3, formula (2.4)], the Laplace transform of $U T$ can be written as

$$
E e^{-t U T}=E \frac{1-e^{-t T}}{t T}=E \frac{1}{t U+1}=\frac{\log (t+1)}{t}, \quad t \geq 0 .
$$

On the other hand, let $R$ be a random variable taking values in $[0,1]$, independent of the uniform empirical process $\left(S_{j}(t), 0 \leq t \leq 1\right), j \in \mathbb{Z}_{+}$, defined 
in (2.13). For any function $f: \mathbb{Z}_{+} \rightarrow \mathbb{R}$, it has been shown in $[5$, Theorem 1$]$ the finite expansion

$$
E f\left(S_{j}(R)\right)=\sum_{m=0}^{j}\left(\begin{array}{c}
j \\
m
\end{array}\right) \Delta^{m} f(0) E R^{m}, \quad j \in \mathbb{Z}_{+} .
$$

With the preceding ingredients, we give the following.

Theorem 3.2. For any $n \in \mathbb{Z}_{+}$, we have

$$
\gamma_{n}=-\frac{1}{n+1} \sum_{k=0}^{n+1}\left(\begin{array}{c}
n+1 \\
k
\end{array}\right) B_{n+1-k}(\log 2)^{n-k} \alpha_{k},
$$

where $\alpha_{k}$ is computed, for each $k \in \mathbb{N}$, as

$$
\alpha_{k}=\sum_{j=1}^{\infty}(-1)^{j} \Delta^{j} \psi_{k}(0) r(j), \quad \psi_{k}(x)=\log ^{k}(x+1), \quad x \geq 0 .
$$

Moreover, we have

$$
\lim _{j \rightarrow \infty}(-1)^{j} \Delta^{j} \psi_{k}(0)=0, \quad k \in \mathbb{N} .
$$

Proof. The proof of (3.7) is given in Coffey [13]. Indeed, using (3.1), (3.2), and the well known formula $\eta(z)=\left(1-2^{-(z-1)}\right) \zeta(z)$, we can write for $|z-1|<1$

$$
(z-1) \zeta(z)=\eta(z) \frac{z-1}{1-e^{-(z-1) \log 2}}=\eta(z) \sum_{k=0}^{\infty} \frac{(-1)^{k} B_{k}}{k !}(\log 2)^{k-1}(z-1)^{k},
$$

and then, by equating the coefficients, we get (3.7). On the other hand, consider the function

$$
\phi_{k}(j)=(-1)^{j} \Delta^{j} \psi_{k}(0), \quad j \in \mathbb{Z}_{+} \quad\left(\phi_{k}(0)=0\right) .
$$

It can be easily checked that

$$
\Delta^{m} \phi_{k}(0)=(-1)^{m} \psi_{k}(m), \quad m \in \mathbb{Z}_{+} .
$$

We therefore have from (1.3), (3.3), and (3.4)

$$
\begin{aligned}
\alpha_{k} & =\sum_{m=0}^{\infty}(-1)^{m} \psi_{k}(m) E U^{m}=\sum_{m=0}^{\infty} \Delta^{m} \phi_{k}(0) E U^{m} \\
& =\sum_{j=0}^{\infty} \phi_{k}(j) E \frac{U^{j}}{(U+1)^{j+1}}=\sum_{j=1}^{\infty}(-1)^{j} \Delta^{j} \psi_{k}(0) r(j),
\end{aligned}
$$

thus showing (3.8).

For the rest of the proof, fix $k \in \mathbb{N}$. Consider the functions

$$
g(j)=j^{k}, \quad f(j)=(-1)^{j} \Delta^{j} g(0), \quad j \in \mathbb{Z}_{+} .
$$

As above, it can be checked that

$$
\Delta^{m} f(0)=(-1)^{m} g(m), \quad m \in \mathbb{Z}_{+} .
$$

Let $\left(U_{i}\right)_{i \geq 1}$ and $\left(T_{i}\right)_{i \geq 1}$ be two sequences of independent identically distributed random variables having the uniform distribution on $[0,1]$ and the 
exponential density $\rho(\theta)=e^{-\theta}, \theta \geq 0$, respectively. Assume that $\left(U_{i}\right)_{i \geq 1}$ and $\left(T_{i}\right)_{i \geq 1}$ are mutually independent and denote by

$$
R=e^{-\left(U_{1} T_{1}+\cdots+U_{k} T_{k}\right)} .
$$

It follows from (3.5) that

$$
E R^{m}=\frac{\log ^{k}(m+1)}{m^{k}}, \quad m \in \mathbb{Z}_{+} .
$$

We thus have from (3.6), (3.10), and (3.11)

$$
\begin{aligned}
E f\left(S_{j}(R)\right) & =\sum_{m=0}^{j}\left(\begin{array}{c}
j \\
m
\end{array}\right)(-1)^{m} g(m) E R^{m} \\
& =\sum_{m=0}^{j}\left(\begin{array}{c}
j \\
m
\end{array}\right)(-1)^{m} \psi_{k}(m)=(-1)^{j} \Delta^{j} \psi_{k}(0), \quad j \in \mathbb{Z}_{+} .
\end{aligned}
$$

Denote by $A=\max \{|f(j)|, j=0, \ldots, k\}$ and observe that $f(j)=0, j>k$. We therefore have from $(3.12)$

$$
\left|(-1)^{j} \Delta^{j} \psi_{k}(0)\right| \leq E\left|f\left(S_{j}(R)\right)\right| \leq A P\left(S_{j}(R) \leq k\right), \quad j \in \mathbb{Z}_{+} .
$$

This shows (3.9), since $S_{j}(R) \rightarrow \infty$, almost surely, as $j \rightarrow \infty$. The proof is complete.

By Lemma 3.1, Theorem 3.2 allows us to compute each $\alpha_{k}$ at the geometric rate $1 / 2$. Thanks to Theorem 3.2, we can evaluate each Stieltjes constant $\gamma_{n}$ at the same geometric rate. In doing this, we need to compute forward differences of powers of logarithms $\Delta^{j} \psi_{k}(0)$, and this can be achieved using Theorem 2.3 in Section 2. A similar result to Theorem 3.2 was obtained by Coffey [13], where each $\alpha_{k}$ is computed at the geometric rate $1 / 3$, with coefficients growing as a polynomial of degree $k$ (see also [3] for slightly different computation formulas). Recently, Johansson [19] has given efficient algorithms to evaluate $\gamma_{n}$ based on the Euler-Mclaurin formula.

\section{The Euler-Mascheroni constant}

The special case of the Euler-Mascheroni constant deserves a more detailed attention. In fact, this constant appears in many different mathematical areas (see, in this regard, the recent survey paper by Lagarias [21]). As follows from Theorem 3.2, to compute this constant we need to evaluate forward differences of the logarithm function. As we will see, such differences can be estimated using formula (3.5) and fastly computed with the help of Theorem 2.3. To this end, note that

$$
(-1)^{j-1} \Delta^{j} \psi_{1}(0)=-\sum_{k=0}^{j}\left(\begin{array}{l}
j \\
k
\end{array}\right)(-1)^{k} \log (k+1)=\log \left(1+P_{j}\right), \quad j \in \mathbb{N},
$$


where

$$
P_{j}=\frac{(\text { odd }) \prod_{k=0}^{j}(k+1)^{\left(\begin{array}{l}
j \\
k
\end{array}\right)}-(\text { even }) \prod_{k=0}^{j}(k+1)^{\left(\begin{array}{l}
j \\
k
\end{array}\right)}}{(\text { even }) \prod_{k=0}^{j}(k+1)^{\left(\begin{array}{l}
j \\
k
\end{array}\right)}}, j \in \mathbb{N},
$$

where (odd) $\prod$ (respectively, (even) $\prod$ )means that the product is extended to those odd (respectively, even) integers $k$ running from 0 to $j$. Observe that $P_{1}=1, P_{2}=1 / 3$, and $P_{3}=5 / 27$.

On the other hand, interchanging in (3.5) the forward difference and the expectation operators, we get

$$
\Delta^{j} \psi_{1}(k)=(-1)^{j-1} E\left(1-e^{-T}\right)^{j} \frac{e^{-k T}}{T}, \quad j \in \mathbb{N}, \quad k \in \mathbb{Z}_{+} .
$$

Observe that the Laplace transform of a random variable $U$ uniformly distributed on $[0,1]$ is given by

$$
E e^{-\theta U}=\frac{1-e^{-\theta}}{\theta}, \quad \theta \geq 0
$$

By (4.1), (4.3), and (4.4), we have for any $j \in \mathbb{N}$

$$
\begin{aligned}
& \log \left(1+P_{j}\right)=E \frac{1}{T}\left(1-e^{-T}\right)^{j}=\int_{0}^{\infty} \frac{\left(1-e^{-\theta}\right)^{j}}{\theta} e^{-\theta} d \theta \\
& =\int_{0}^{\infty} E e^{-\theta U}\left(1-e^{-\theta}\right)^{j-1} e^{-\theta} d \theta=\frac{1}{j} \int_{0}^{\infty} E U e^{-\theta U}\left(1-e^{-\theta}\right)^{j} d \theta,
\end{aligned}
$$

where the last equality follows by integration by parts. We give the following auxiliary result which may have interest by itself.

Lemma 4.1. The sequence $\left(P_{j}\right)_{j \geq 1}$ defined in (4.2) strictly decreases from $P_{1}=1$ to 0 . In addition, for any $j=2,3, \ldots$, we have

$$
\left(1-\frac{1}{j}\right)^{j+1} \frac{1}{j \log j} \leq \log \left(1+P_{j}\right) \leq \frac{e}{j \log j}+\frac{1}{j} \exp \left(-j^{1-1 / e}\right) .
$$

As a consequence,

$$
P_{j} \sim \frac{e}{j \log j}, \quad \text { as } \quad j \rightarrow \infty .
$$

Proof. The first statement is an immediate consequence of (4.5). Using Fubini's theorem and (4.4), we have

$$
\begin{aligned}
& \int_{\log j}^{\infty} E U e^{-\theta U}\left(1-e^{-\theta}\right)^{j} d \theta \geq\left(1-\frac{1}{j}\right)^{j} \int_{\log j}^{\infty} E U e^{-\theta U} d \theta \\
& =\left(1-\frac{1}{j}\right)^{j} E e^{-U \log j}=\left(1-\frac{1}{j}\right)^{j+1} \frac{1}{\log j} .
\end{aligned}
$$


This, together with (4.1) and (4.5) shows the lower inequality in (4.6). On the other hand, using the inequality $1-x \leq e^{-x}, x \geq 0$, we have

$$
\begin{aligned}
& \int_{0}^{e^{-1} \log j} E U e^{-\theta U}\left(1-e^{-\theta}\right)^{j} d \theta \\
& \leq\left(1-\frac{1}{j^{1 / e}}\right)^{j} \int_{0}^{e^{-1} \log j} E U e^{-\theta U} d \theta \leq \exp \left(-j^{1-1 / e}\right) .
\end{aligned}
$$

Finally, we see from (4.4) that

$$
\begin{aligned}
\int_{e^{-1} \log j}^{\infty} E U e^{-\theta U}\left(1-e^{-\theta}\right)^{j} d \theta & \leq \int_{e^{-1} \log j}^{\infty} E U e^{-\theta U} d \theta \\
& =E e^{-U e^{-1} \log j} \leq \frac{e}{\log j} .
\end{aligned}
$$

This, in conjunction with (4.7), shows the upper inequality in (4.6) and completes the proof.

Finally, we give the following result to compute the Euler-Mascheroni constant.

Theorem 4.2. For any $n \in \mathbb{N}$, we have

$$
\gamma_{0}-\frac{\log 2}{2}-\frac{1}{\log 2} \sum_{j=1}^{n} r(j) \log \left(1+P_{j}\right)<\frac{1}{\log 2} \frac{\log \left(1+P_{n+1}\right)}{(n+2) 2^{n}},
$$

where $r(j)$ and $P_{j}$ are defined in Lemma 3.1 and (4.2), respectively.

Proof. Since $B_{0}=1$ and $B_{1}=-1 / 2$, we have from (3.7)

$$
\gamma_{0}=\frac{\log 2}{2}-\frac{\alpha_{1}}{\log 2}=\frac{\log 2}{2}+\frac{1}{\log 2} \sum_{j=1}^{\infty} r(j) \log \left(1+P_{j}\right),
$$

where we have used (3.8) and (4.1) in the last equality. Hence, the conclusion follows from (4.8) and Lemmas 3.1 and 4.1.

Theorem 4.2 provides a monotone and fast computation of Euler's constant $\gamma_{0}$. In turn, the coefficients $\log \left(1+P_{j}\right), j \in \mathbb{N}$, can be fastly computed as (recall Theorem 2.3)

$$
\log \left(1+P_{j}\right)=\frac{4\left(P_{j}+2\right)}{P_{j}} \sum_{k=0}^{\infty}(-1)^{k} c(k)\left(\frac{P_{j}^{2}}{P_{j}^{2}+8\left(P_{j}+1\right)}\right)^{k+1} .
$$

Observe that, by virtue of Lemma 4.1, the series in (4.9) has a geometric rate of the orden of

$$
\frac{e^{2}}{e^{2}+8 e j \log j+8(j \log j)^{2}}, \quad j=2,3, \ldots
$$

Using continued fractions, Lu et al. [23, 24] have obtained monotone convergence to $\gamma_{0}$ of the order of $O\left(n^{-p}\right), p=3,4,5$ (see also $\mathrm{Lu}$ [22] and Yang [25]). Kh. and T. Hessami Pilehrood [18] have constructed a rational 
approximation $p_{n} / q_{n}$ converging to $\gamma_{0}$ subexponentially. Series representations for $\gamma_{0}$ with an exponential rate of decay can be found in Coffey $[12,14]$. Finally, a fast computation of $\gamma_{0}$ with geometric rate $1 / 3$ is provided in Coffey [13].

\section{Acknowledgment}

The authors would like to thank the referees for their careful reading of the manuscript and for their remarks and suggestions, which greatly improved the final outcome.

\section{References}

[1] Abramowitz, M., Stegun, I. 1972 Handbook of Mathematical Functions. Dover, New York.

[2] Adell, J.A. 2011. Asymptotic estimates for Stieltjes constants: a probabilistic approach. Proc. R. Soc. Lond. Ser. A Math. Phys. Eng. Sci. 467, no. 2128, 954-963.

[3] Adell, J.A. 2012. Estimates of generalized Stieltjes constants with a quasigeometric rate of decay. Proc. R. Soc. Lond. Ser. A Math. Phys. Eng. Sci. 468, no. 2141, 1356-1370.

[4] Adell, J. A. 2013. Differential calculus for linear operators represented by finite signed measures and applications. Acta Math. Hungar. 138, no. 1-2, 44-82.

[5] Adell, J.A., Anoz, J.M. 2008. Signed binomial approximation of binomial mixtures via differential calculus for linear operators. J. Statist. Plann. Inference, 138, no. 12, 3687-3695.

[6] Adell, J.A., Lekuona, A. 2010. Rates of convergence for the iterates of Cesàro operators. Proc. Amer. Math. Soc. 3, no. 3, 1011-1021.

[7] Adell, J.A., Lekuona, A. 2015. Dirichlet's eta and beta functions: concavity and fast computation of their derivatives. J. Number Theory 157, 215-222.

[8] Alzer, H., Kwong, M.K. 2015. On the concavity of Dirichlet's eta function and related functional inequalities. J. Number Theory 151, 172-196.

[9] Berndt, B.C. 1972. On the Hurwitz zeta-function. Rocky Mountain J. Math. 2, 151-157.

[10] Borisov, B., Shkodrov, V. 2007. Divergent series in the generalized binomial transform.Adv. Stud. Contemp. Math. (Kyungshang) 14, no. 1, 77-82.

[11] Borwein, P. 2000. An efficient algorithm for the Riemann zeta function. In Constructive, experimental, and nonlinear analysis (Limoges, 1999), CMS Conf. Proc., Amer. Math. Soc., Providence, RI, 27, 29-34.

[12] Coffey, M.W. 2009. An efficient algorithm for the Hurwitz zeta and related functions. J. Comput. Appl. Math. 225, no. 2, 338-346.

[13] Coffey, M.W. 2010. The Stieltjes constants, their relation to the $\eta_{j}$ coefficients, and representation of the Hurwitz zeta function. Analysis (Munich) 30, no. 4, 383-409.

[14] Coffey, M.W. 2014. Series representations for the Stieltjes constants. Rocky Mountain J. Math. 44, no. 2, 443-477. 
[15] Cohen, H., Rodriguez Villegas, F., Zagier, D. 2000. Convergence acceleration of alternating series. Experiment. Math. 9, no. 1, 3-12.

[16] Flajolet, P., Vepstas, L. 2008. On differences of zeta values. J. Comput. Appl. Math. 220, no. 1-2, 58-73.

[17] Hessami Pilehrood, Kh., Hessami Pilehrood, T. 2010. Series acceleration formulas for beta values. Discrete Math. Theor. Comput. Sci. 12, no. 2, 223-236.

[18] Hessami Pilehrood, Kh., Hessami Pilehrood, T. 2013. On a continued fraction expansion for Euler's constant. J. Number Theory 133, no. 2, 769-786.

[19] Johansson, F. 2015. Rigorous high-precision computation of the Hurwitz zeta function and its derivatives. Numer. Algorithms 69, no. 2, 253-270.

[20] Karatsuba, E.A. 2013. Fast calculation of the Catalan constant via approximations obtained by Kummer-type transformations. Diskret. Mat. 25, no. 4, $74-87$.

[21] Lagarias, J.C. 2013. Euler's constant: Euler's work and modern developments. Bull. Amer. Math. Soc. (N.S.) 50, no. 4, 527-628.

[22] Lu, D. 2014. Some quicker classes of sequences convergent to Euler's constant. Appl. Math. Comput. 232, 172-177.

[23] Lu, D., Song, L., Yu, Y. 2015. Some new continued fraction approximation of Euler's constant. J. Number Theory 147, 69-80.

[24] Lu, D., Song, L., Yu, Y. 2015. New sequences with continued fraction towards Euler's constant. Appl. Math. Comput. 259, 12-20.

[25] Yang, S. 2012. On an open problem of Chen and Mortici concerning the EulerMascheroni constant. J. Math. Anal. Appl. 396, no. 2, 689-693.

\author{
José A. Adell \\ Departamento de Métodos Estadísticos \\ Facultad de Ciencias \\ Universidad de Zaragoza \\ 50009 Zaragoza \\ Spain \\ e-mail: adell@unizar.es \\ Alberto Lekuona \\ Departamento de Métodos Estadísticos \\ Facultad de Ciencias \\ Universidad de Zaragoza \\ 50009 Zaragoza \\ Spain \\ e-mail: lekuona@unizar.es
}

